\title{
Machine Learning based Identification and Classification of Disorders in human Knee Joint - Computational Approach
}

\section{Balajee A. ( $\sim$ balajeese7en@gmail.com )}

SASTRA Deemed University: Shanmugha Arts Science Technology and Research Academy

\section{Venkatesh Rajagopal}

SASTRA Deemed University: Shanmugha Arts Science Technology and Research Academy

\section{Research Article}

Keywords: Computation, Vibroarthrographic (VAG) signal, Greedy mutual informative regressed sequential backward selection algorithm (GSBFS), dichotomous logit regression, Radial basis kernelized least square support vector classifier (RKLSSVC)

Posted Date: June 15th, 2021

DOl: https://doi.org/10.21203/rs.3.rs-591767/v1

License: (a) (1) This work is licensed under a Creative Commons Attribution 4.0 International License. Read Full License

Version of Record: A version of this preprint was published at Soft Computing on August 18th, 2021. See the published version at https://doi.org/10.1007/s00500-021-06134-0. 


\title{
MACHINE LEARNING BASED IDENTIFICATION AND CLASSIFICATION OF DISORDERS IN HUMAN KNEE JOINT - COMPUTATIONAL APPROACH
}

\author{
A. Balajee ${ }^{1, *}$ and R. Venkatesan ${ }^{2}$ \\ ${ }^{1}$ School of Computing, SASTRA Deemed University, Thanjavur, Tamil Nadu, India \\ ${ }^{2}$ Department of Information Technology, School of Computing, SASTRA Deemed University, \\ Thanjavur, Tamil Nadu, India
}

*Corresponding author: venkatesan@it.sastra.edu

\begin{abstract}
Earlier identification of knee joint pathology helps the therapist to provide the appropriate clinical procedures to control the deteriorating process of arthritis. Beyond usual medical investigations, computational techniques have been used for the diagnosis of knee joint disorder. Among different methodologies, Vibroarthrographic technique is employed to identify knee joint disorder. Machine Learning contains number of classification methods for the given data. A novel technique called Greedy sequential backward feature selection based Radial kernelized least square support vector classification (GSBFS-RKLSSVC) is introduced for accurate detection of knee joint pathology with minimum time. The proposed GSBFS-RKLSSVC technique consists of three processes namely feature selection, feature evaluation and classification. Initially number of VAG signal images are taken from the dataset for detection of knee joint disorder. The relevant feature is selected through the Greedy mutual informative regressed sequential backward selection algorithm to reduce an initial dimensional feature space into a low dimensional feature subspace. Following this the dichotomous logit regression is applied to select the best features and discard others. Therefore, the feature selection process of the proposed GSBFS-RKLSSVC minimizes the time consumption of the knee joint pathology detection. Once the signal features are extracted, RKLSSVC is applied to detect the normal and abnormal VAG signal. Decision boundary is utilized by the classifier to categorize the samples based on the similarity between the training features and testing features. As a result, the accurate classification is obtained with a minimum error rate. The observed result indicates that GSBFS-RKLSSVC achieves higher accuracy, sensitivity, specificity and reduces time than the conventional methods.
\end{abstract}


Keywords: Computation, Vibroarthrographic (VAG) signal, Greedy mutual informative regressed sequential backward selection algorithm (GSBFS), dichotomous logit regression, Radial basis kernelized least square support vector classifier (RKLSSVC)

\section{Introduction}

The human knee joint is complex and a main joint in the human body. It offers flexible movements and maintains weight of body. But the human knee joint bears excess of load and it is highly prone to injuries. Therefore, the early detection of knee joint injuries helps the physician provide appropriate treatment. Recently, the various diagnosis methods of joint disorders have been done using image based techniques including X-ray. X-rays lack in providing a better performance measure. Vibroarthrographic (VAG) signal is a recently emerged methodology for pathology detection in human knee joints. The VAG signal based knee joint disorder prediction provides better accuracy.

Athavale and Krishnan (2020), a unique actigraphy-based VAG signal analysis system was introduced for identifying the knee-joint conditions through the feature extraction adaptive segmentation. The designed system improves the accuracy, sensitivity, and specificity. But, the time complexity of knee-joint disorder identification was not minimized. An Optimal Bandwidth-Duration Localized Three-Band Orthogonal Wavelet Filter Banks (OBDLTBOWFB) was introduced in Sharmaa and Acharya (2018) for early detection of the normal kneejoints using VAG signals. The designed method failed to apply the large VAG signal sample images acquired from more subjects.

To minimize the time complexity of knee-joint disorder diagnosis, better features were extracted in Befrui et al. (2018). However, the designed method failed to integrate both temporal and frequency features to improve the accuracy. The vibroarthrographic evaluation of changes in arthrokinematics was analyzed in Ołowiana et al. (2020) with variated loads and correlation between the features. But an efficient technique was not implemented to find exact features for minimizing the complexity. 
A computer-aided diagnostic (CAD) system was developed in Nalband et al. (2018) for knee-joint disorders detection through the time-frequency analysis. But the designed CAD system failed to diagnose the knee joint disorders. Novel descriptors were developed in Łysiak et al. (2016) for the initial diagnosis of the healthy knee, and different stages of osteoarthritis by extracting the VAG vibroarthrographic signals. But the sensitivity and specificity analysis were not performed. A 5-Class Knee Joint Classification was performed in Adam et al. (2020) based on the VAG Signal Spectral Features. Through the classification method increases the accuracy, the time consumption was not minimized.

An investigation of characteristics and spatial changes of VAG activity with the effect of the knee extension and flexible movements using dissimilar added loads was performed in Andersen, Arendt-Nielsen, and Madeleine (2018). But the feature selection and classification techniques were not applied to find knee joint disorder. The reliability of Knee joint sound signals was analyzed in Kalo et al. (2020a) using Vibroarthrography during the movement conditions.

A pathological knee joint was detected in Kręcisz and Bączkowicz (2018) using VAG signals by extracting the various features. But the algorithms failed to apply new methods for signal analysis in categorization of VAG signals gathered from joints.

Considering the afore-mentioned inefficiencies in VAG signal analysis, a novel technique called GSBFS-RKLSSVC is introduced in this paper. The major contribution of the GSBFSRKLSSVC technique is summarized as given below,

* To increase the detection accuracy of knee joint disorder, a novel GSBFS-RKLSSVC technique is introduced based on feature selection and classification.

To minimize the time complexity, a Greedy mutual informative regressed sequential backward selection algorithm is designed. The mutual information is measured to find the relevant features for knee joint disorder. Then the dichotomous logit regression is applied to select the best features and discard the other features.

* A radial basis kernelized least square support vector classifier is employed in GSBFSRKLSSVC to find the normal and abnormal VAG signal based on the selected relevant features. The classifier uses the radial basis kernel function to measure the similarity 
between the testing and training features for identifying the normal and abnormal signal with minimum error.

Finally, experiments were conducted with various algorithms to find the performance of the GSBFS-RKLSSVC technique based on various metrics.

\subsection{Organization of the paper}

The initial section was an introduction. The second one discusses the related works. Section three provides a brief description of the GSBFS-RKLSSVC technique. Section four provides an experimental description of the VAG signal image dataset. Section five provides a discussion of the quantitative results of different parameters. Finally, in section six, the conclusion is presented.

\section{Related works}

The potential of non-invasive identification of knee osteoarthritis was performed in (Yiallourides and Naylor 2020) using the VAG signal image during the walking conditions. But the accuracy of detection of knee osteoarthritis was not higher. Knee joint sounds were detected in Kalo et al. (2020b) based on the knee joint loading conditions using VAG signal. A Support Vector Machines (SVM) classifier was developed in Fredo (2017) to categorize normal and KJD vibroarthrographic (VAG) signals. However, the inability to classify the different stages was recorded.

A Kohonen neural network was introduced in Nouma, Mitiche and Mezghani (2019) for knee pathologies classification. But the time complexity analysis was not performed. An Ensemble Empirical Mode Decomposition model was developed in Nalband, Sreekrishna and Prince (2016) for classifying the Knee Joint Vibration Signals based on the entropy-based feature extraction. But the higher accuracy was not attained.

A different machine learning technology was introduced in Zheng et al. (2020) for classifying the knee joint vibration signal used for the clinical diagnosis of knee joint diseases. However, the algorithm did not focus to reduce the time complexity of disease diagnosis. Spatial dependencies were evaluated in Madeleine et al. (2020) through the multi-channel recordings of 
knee osteoarthrosis patients. But it failed to analyze the other time domain and complexity features for identifying knee osteoarthrosis.

A method that integrates the empirical mode decomposition (EMD) and wavelet transform was introduced in Gong et al. (2020) to diagnose knee osteoarthritis by recording the knee vibroarthrographic (VAG) signals. However, it was difficult to perform the early-stage diagnosis. Multichannel VAG signals were employed in Samani et al. (2020) to classify the knee osteoarthritis patients with higher sensitivity. But the complexity analysis remained unsolved. Kellgren-Lawrence (KL) grade system was developed in Shieh et al. (2016) for recording the vibroarthrographic signals to identify knee Osteoarthritis. However, accurate knee osteoarthritis detection was not performed.

\section{Proposal methodology}

In this section, the proposed GSBFS-RKLSSVC technique is developed for accurate VAG signals classification and knee joint disorder detection with higher accuracy and minimum time. The GSBFS-RKLSSVC consists of feature extraction and classification. In general, the VAG signals image consists of multiple features, reduces the performance of classification and it also takes more processing time. Therefore, significant feature selection is a major role in accurate VAG signal classification. In addition, significant feature selection is more efficient for reducing the computation time of VAG signal classification.

The architecture of GSBFS-RKLSSVC technique is depicted in Figure 1 with different processing steps namely VAG signal image collection, feature selection, evaluation, and classification. The numbers of VAG signal images are collected from the database $s_{1}, s_{2}, s_{3} \ldots s_{n}$. Then the next process of the proposed GSBFS-RKLSSVC technique is to perform the feature selection through the Greedy Mutual Informative Regressed Sequential Backward Selection (GMIRSBS) algorithm. This is followed by the feature valuation which is done with the help of the Dichotomous logit (i.e. logistic) regression. Finally, the classification is done with the extracted signal features using Radial Basis Kernelized Least Square Support Vector Classifier (RBKLSSVC). 


\subsection{Greedy mutual informative regressed sequential backward selection}

The first process in GSBFS-RKLSSVC is to perform feature selection from VAG signal image. The VAG signal consists of multiple time and frequency domain features, complexity features, and nonlinear features. While considering the entire features, it takes more complexity in classification. Therefore, more relevant features are chosen to reduce the complexity in classification. Feature selection is a major concern since it helps to minimize the errors from the irrelevant features. The proposed GSBFS-RKLSSVC technique uses the GMIRSBS algorithm for selecting the best feature and discards the word features based on the mutual information.

Figure 2 given above illustrates the flow process of relevant feature selection and worst features selection. The features extracted from the VAG signal are time and frequency domain features, complexity features, and nonlinear features. VMS is mean-squared values of obtained signal, R4 indicates signal amplitude measured as difference among mean of four maximum and mean of four minimum values. F470, F780, P1, P2 are spectral feature vectors. Complexity features are FF (Form factor), SHE (Shannon entropy), TC (turns count), DFA (fractal scaling index), MSE (multiscale sample entropy). The non linear features are RR, DET (determinism), LAM (laminarity score), ENT (entropy), TT (trapping time), LMAX (max line).

Consider number of features obtained from VAG signal is expressed in below equations

$$
F=\left\{f_{1}, f_{2}, f_{3}, \ldots f_{n}\right\} \in \text { VAG signal }
$$

From (1), $F$ is set of original features $f_{1}, f_{2}, f_{3}, \ldots f_{n}$ in VAG signal. Among several features, Greedy mutual informative sequential backward selection begins with original feature set. For each iteration, mutual information gain is computed between the features and objective (i.e. knee joint disorder).

The mutual information of two random variables is used to find the mutual dependence between them.

$$
M D(F, O)=\log _{2}\left(\frac{P(F, O)}{P(F) P(O)}\right)
$$

where, $M D(F, O)$ denotes a mutual dependence, $F$ denotes a set of original features, $O$ represents the objective function, $P(F, O)$ represents the joint probability distribution of $F$ and $\mathrm{O}, P(F) P(O)$ symbolizes the marginal probability distribution of $F$ and $O$, respectively. 
The proposed feature selection algorithm also uses the dichotomous logit (i.e. logistic) regression to evaluate the features based on the mutual information. The regression is a statistical method to analyze the mutual information gain value and produces dichotomous outcomes (i.e. Two possible outcomes) coded as 1 (select best features with maximum information gain score) or 0 (discard the worst features with minimum information gain).

Figure 3 shows the Dichotomous logit Regression analysis to identify the best features and discard the worst features. After that, the best feature is selected based on the maximum mutual information between the feature set and objective function.

$$
f(x)=\left\{\begin{aligned}
& 1 ; \text { if } \arg \max \{M D(F, O)\} \\
& 0 ; \text { otherwise }
\end{aligned}\right.
$$

where ' $f(x)$ ' denotes an output of regression function, arg max denotes an argument of the maximum function to maximize the mutual information $M D$. The sequential backward selection is to sequentially discard the features from the given features list and to reach the list of minimum best features. At every step of removal, the feature that causes the least performance loss gets removed. In this way, the relevant feature subset is obtained. 


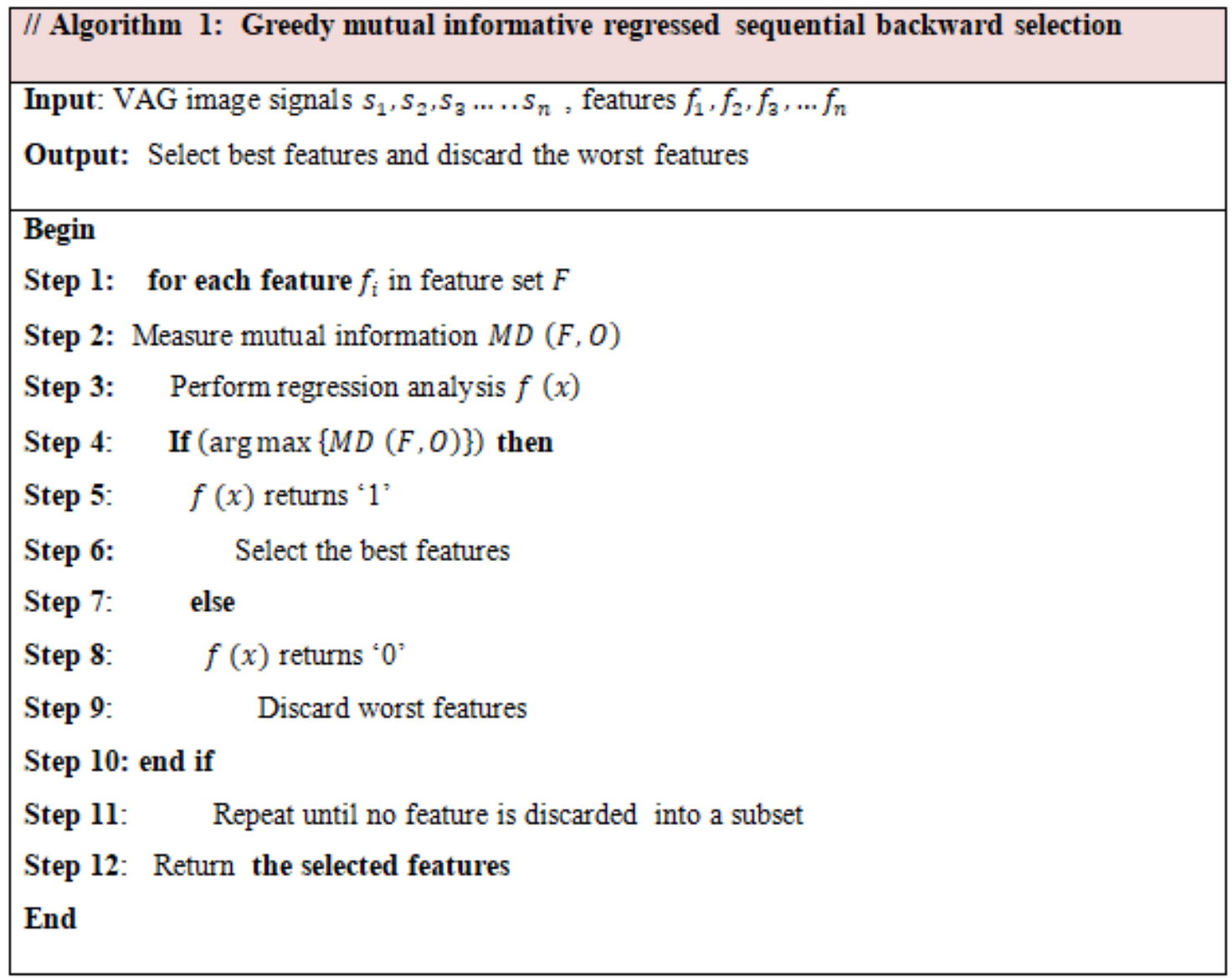

Algorithm 1 given above describes the Greedy mutual informative regressed sequential backward selection algorithm. The number of features is extracted from the VAG signal image. Then the extracted features are given to the sequential backward selection algorithm to sequentially remove the features and select the best features. The best and worst features are identified based on the regression analysis. The Dichotomous logit regression function analyzes the mutual information gain between the features and objectives. The maximum mutual dependence is used to select the relevant best features. Otherwise, the worst feature gets discarded from the feature list. Thus, GMIRSBS chooses relevant features for accurate detection of knee joint disorder with minimum time complexity.

\subsection{Radial basis kernelized least square support vector classifier}

The proposed GSBFS-RKLSSVC performs the early detection of knee joint pathology using a Radial basis kernelized least square support vector classifier (RBKLSSVC) with the 
selected feature extracted from the VAG signal. The proposed RBKLSSVC is a type of supervised learning that categorizes the input VAG signal images into normal or abnormal (i.e knee joint pathology). The proposed technique uses a Radial basis kernel for measuring the similarity between any pair of inputs. The least-square is the process of minimizing the difference of the squares between an observed value and predicted value obtained from the classification process. This is also called an error. Therefore, the proposed RBKLSSVC technique improves the accuracy of knee joint disorder detection and minimizes the error.

Let us consider the raining samples $\left\{\left(f_{1}, y_{1}\right),\left(f_{2}, y_{2}\right), \ldots\left(f_{n}, y_{n}\right)\right\}$ where $f_{n}$ denotes selected features and $y_{i}$ represents the outcomes whose value is found by observation. The outcomes variable provides the two label as $y_{i} \in\{+1,-1\}$ where the output ' $y_{i}=+1$ 'is a normal, and the output $y_{i}=-1$ is abnormal. Therefore, the normal or category image samples are obtained by using a separating hyperplane. The construction of RBKLSSVC is shown in Figure 4.

Figure 4 given above illustrates the classification process of the Radial basis kernelized least square support vector. As shown in Figure 4, the separating hyperplane $(\varphi)$ is used as a decision boundary to categorize the given input samples either upper or lower. The classifier also uses the two support vectors for the upper and lower side of hyperplane. The upper sides of categorized samples are termed normal whereas lower labeled samples are abnormal. These two classes are obtained by using the following mathematical equations.

A separating hyperplane is written as a set of the input satisfying the following equation

$$
\boldsymbol{\varphi} \rightarrow w \cdot f+c=0
$$

where, $\boldsymbol{\varphi}$ denotes a hyperplane (i.e. decision boundary), $f$ denotes training samples (i.e. selected features), ' $c$ ' indicates a bias and $w$ denotes a normal weight vector to the hyperplane $(\varphi)$. When training samples are linearly separable, two parallel support vectors are chosen which separate two classes $(+1$ and -1$)$. Therefore, the input that belongs to the above and below side of the decision boundary is formulated as follows:

$$
\begin{aligned}
& z_{1} \rightarrow w \cdot f+c>0 \quad \text { (Above the boundary is of one class, with label 1) } \\
& z_{2} \rightarrow w \cdot f+c<0 \quad \text { (Below the boundary is of one class, with label -1) }
\end{aligned}
$$


where, $z_{1}, z_{2}$ denotes lower and upper support vectors to categorize the images as above and below the boundary. The sample classified above the boundary is called normal and below the boundary is called abnormal. The predicted output $\left(\boldsymbol{y}^{\prime}\right)$ of the classifier with the kernel function is given below:

$$
Y=\operatorname{sign} \sum w y_{i} \beta\left(f_{i}, f_{n}\right)
$$

where, $\boldsymbol{Y}$ represents predicted classification results, "sign" denotes whether predicted classification as positive or negative, $\beta\left(f_{i}, f_{n}\right)$ indicates kernel function which calculates similarity among selected feature (i.e. training feature) $f_{i}$ and normal knee joint features ' $f_{n}$ ' (i.e. testing feature), $w$ indicates weights of training samples. Here, radial basis kernel function is applied to measure the similarity between the selected feature and the normal knee feature. The radial basis kernel ' $\beta\left(f_{i}, f_{n}\right)$ ' is expressed as follows:

$$
\beta\left(f_{i}, f_{n}\right)=\exp \left(\frac{\left\|f_{i}-f_{n}\right\|^{2}}{d^{2}}\right)
$$

where $d$ indicates a deviation. The feature which is more similar to the normal knee joint features is classified above the decision boundary, hence it is called normal. The features classified below the decision boundary are called abnormal (i.e. knee joint disorder). Based on the kernel measurement, the decision boundary correctly classifies the given input samples. After the classification, the Least-squares function in the proposed classification technique is to minimize the error which is expressed as given below:

$$
\begin{array}{r}
\mathrm{Q} \rightarrow \arg \min E \\
E=\sum_{i=1}^{n}\left(\boldsymbol{Y}_{a}-\boldsymbol{Y}\right)^{2}
\end{array}
$$

From (9) and (10), Q denotes the least square function, ' $E$ ' denotes an error rate, $\boldsymbol{Y}_{a}$ denotes actual classification results, $Y$ denotes a predicted output, arg min denotes an argument of a minimum function to minimizes the error and improve the classification results with minimum time. In this way, knee joint pathology is correctly detected through the classification using a VAG signal image. 


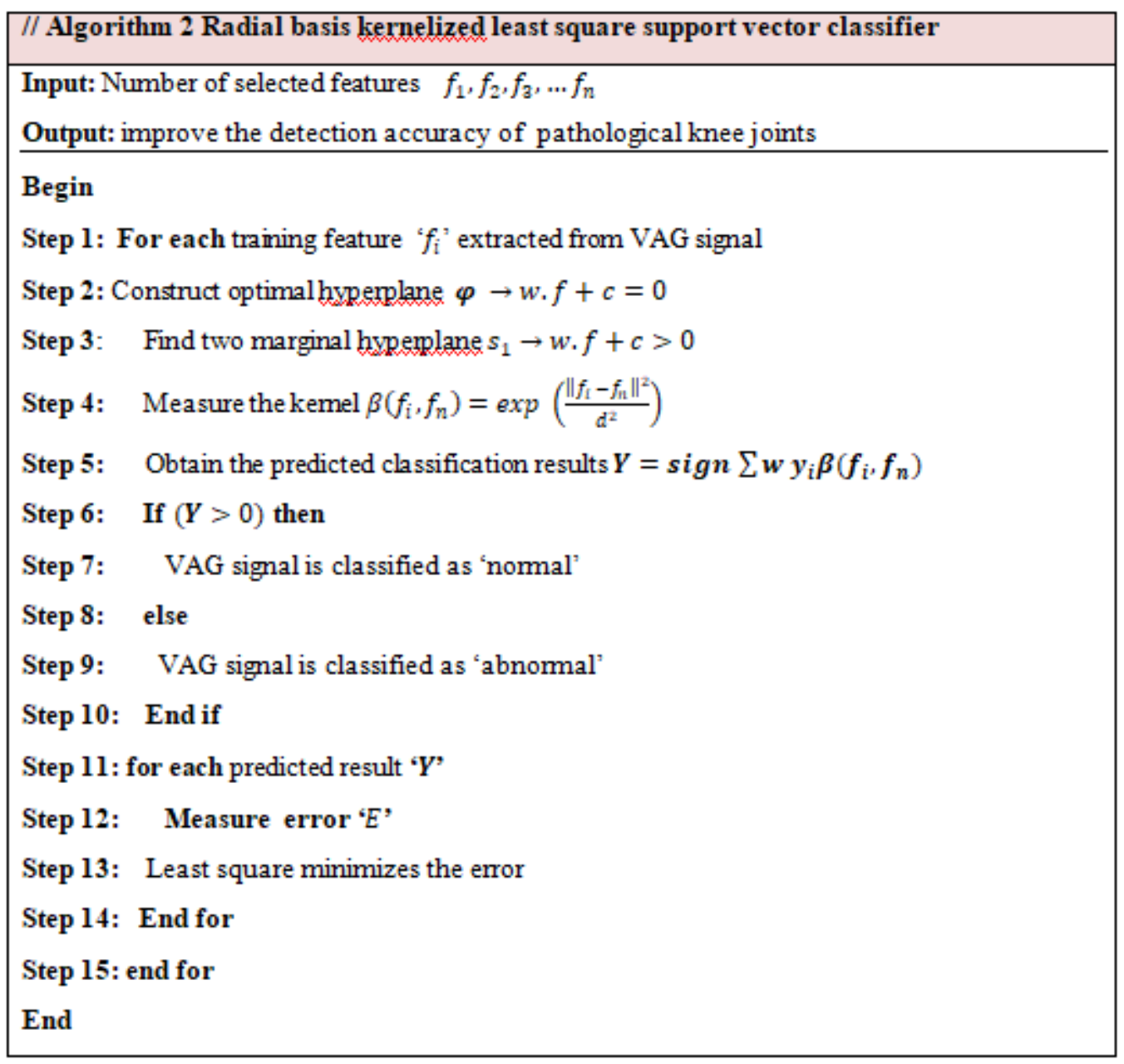

The Algorithm 2 explains the process of Radial basis kernelized least square support vector classifier based normal' and abnormal VAG signal classification. Extracted and selected features are sent to the classifier. The classifier uses the decision boundary to categorize the samples based on the similarity between the training features and testing normal knee joint features. These two features are more similar and the hyperplane classifies the VAG signal into the upper side, i.e., normal. Otherwise, it is classified into the below hyperplane, i.e. abnormal. Based on the results, the proposed technique classifies the VAG signal and the least square function minimizes the training error.

\section{Experimental setup}


Experimental assessment of the proposed GSBFS-RKLSSVC technique and the two existing Athavale and Krishnan (2020) and Sharmaa and Acharya 2018) are implemented using MATLAB coding for detecting the normal or abnormal VAG at an earlier stage. For the experimentation, VAG signal images of samples are collected from the https://people.ucalgary.ca/ ranga/enel563/SIGNAL_DATA_FILES/. Totally 150 VAG signal image samples are collected from both normal and abnormal to measure the performance of our suggested method and existing methods.

Initially, the multiple time and frequency domain features are extracted from the input VAG signal images and the more relevant features are selected by applying the Greedy Mutual informative regressed sequential backward selection algorithm. Finally, the extracted features are given to the Radial basis kernelized least square support vector classifier. The classifier analyzes the extracted features and classifies the VAG signal images into normal or abnormal.

\section{Performance results and discussion}

In this section, quantitative analyses of the proposed GSBFS-RKLSSVC and Athavale and Krishnan (2020) and Sharmaa and Acharya (2018) are discussed with different performance metrics such as accuracy, sensitivity, specificity, and detection time. For the discussion of the quantitative results, VAG signal images are taken from the database

\subsection{Performance analysis of Accuracy}

Accuracy is measured as number of image samples that are correctly detected as a knee joint pathology from given input VAG signal images. The accuracy computation is mathematically expressed as given below

$$
A=\frac{T N+T P}{T N+T P+F N+F P} * 100
$$

where ' $A$ ' denotes an accuracy, $T P$ denotes a true positive, $T N$ indicates a true negative, $F N$ denotes a false negative, FP denotes a false positive. The accuracy is measured It is measured in terms of percentage $(\%)$. 
Table 1 reports the performance analysis of accuracy versus number of VAG signal images ranges from 15 to 150 . For each method, ten outcomes are observed with number of inputs. From the attained outcomes, the GSBFS-RKLSSVC technique achieves higher accuracy than the other two methods. This is validated through the mathematical evaluation. Let us consider 15 VAG signal image samples for calculating the accuracy. By applying the GSBFSRKLSSVC technique, $86.66 \%$ of accuracy is observed whereas the accuracy of existing Athavale and Krishnan (2020) and Sharmaa and Acharya (2018) are $73.33 \%$ and 66.66\%, respectively. Similarly, nine results are obtained and the accuracy of the proposed technique is compared to the existing methods. The average of ten comparison results indicates that the accuracy of the GSBFS-RKLSSVC technique increased by $10 \%$ and $16 \%$ than the conventional methods.

Figure 5 depicts the comparison plot of accuracy using three different methods namely GSBFS-RKLSSVC, Athavale and Krishnan (2020) and Sharmaa and Acharya (2018). The graphical plot shows that the VAG signal images are taken in the ' $x$ ' axis and the classification accuracy is obtained in the ' $y$ ' axis. The accuracy of three different methods is represented by three various colors namely blue, red and green. The graphical plot indicates that the GSBFSRKLSSVC increases the performance of accuracy by applying the Radial basis kernelized least square support vector classifier. The proposed classifier uses the Radial basis kernel function to measure the similarity between the selected features and the testing normal features. Based on the similarity value, the normal' and abnormal VAG signals are classified into either side of the decision boundary with higher accuracy.

\subsection{Performance analysis of sensitivity}

The sensitivity is the ratio of true positive rate to the summation of a truly positive and false negative. The sensitivity is calculated as given below,

$$
\text { Sensitivity }=\frac{T P}{T P+F N} * 100
$$

where ' $T P$ ' denotes a true positive, $F N$ denotes a false negative. The true positive refers to the abnormal samples correctly identified and the false negative refers to the normal samples incorrectly identified as abnormal samples. Sensitivity is measured in terms of percentage (\%). 
Table 2 and Figure 6 given above report the performance comparison of sensitivity analysis of three techniques namely GSBFS-RKLSSVC, Athavale and Krishnan (2020) and Sharmaa and Acharya (2018). The observed results noticed that the GSBFS-RKLSSVC outperforms well in terms of achieving higher sensitivity results than the other existing methods. Let us consider the 15 VAG signal image samples as input, the true positive rate of the GSBFSRKLSSVC technique is 11 and the false-negative rate is 1 , then the sensitivity value is 91.66\%. But the sensitivity values of Athavale and Krishnan (2020) and Sharmaa and Acharya (2018) are $80 \%$ and $77.7 \%$, respectively. Likewise, nine sensitivity results are obtained for each method with a number of VAG signal images. Then the percentages of the sensitivity value of the proposed technique are compared to existing methods. The average results confirm that the GSBFS-RKLSSVC increases the performance of sensitivity by $7 \%$ and $10 \%$ than the state-ofthe-art methods. This is due to the reason of the proposed GSBFS-RKLSSVC technique uses the least square function after the classification to reduce the error rate and it improves the accurate classification of normal or abnormal VAG signal images.

\subsection{Performance analysis of Specificity}

Specificity is defined as the ratio of actual negatives and the summation of true negative and false positives.

$$
\text { Specificity }=\frac{T N}{T N+F P} * 100
$$

where ' $T N$ ' denotes a true negative, FP denotes false positive. It is calculated in percentage (\%).

The performance evaluation of specificity along with the number of input VAG signal image samples in the counts from 10 to 150 are reported in Table 3. The specificity is calculated based on true negatives and false positives. Let us consider the $15 \mathrm{VAG}$ signal image samples for computing the specificity. Among the $15 \mathrm{VAG}$ signal images, the number of true negatives is 2 and the false positives is 1 . Therefore, the specificity of the GSBFS-RKLSSVC is $66.66 \%$. The

specificities of the existing Athavale and Krishnan (2020) and Sharmaa and Acharya (2018) are $60 \%$ and $50 \%$, respectively. Similarly, the specificity is calculated for different VAG signal images. The specificity is increased by $21 \%$ and $26 \%$ as compared to Athavale and Krishnan (2020) and Sharmaa and Acharya (2018), respectively.

Figure 7 depicts the experimental results of specificity versus a number of VAG signal images taken from the datasets. The graphical plot indicates that the GSBFS-RKLSSVC 
technique provides better performance when compared to the conventional methods. Radial basis kernelized least square support vector classifier uses the decision boundary to classify the given samples based on the similarity between the training features and testing normal knee joint features. This helps to minimize the false positives and false negatives. In addition, the least square function reduces the training error and provides an accurate classification.

\subsection{Performance analysis of time complexity}

Time complexity is calculated as the amount of time consumed to discover knee joint disorder based on classification. The overall time consumption is calculated as follows:

$$
T C=n * T(C O S)
$$

where $T C$ indicates a time complexity, $n$ represents the number of samples, $T(C O S)$ indicates a time for classifying one VAG signal image. The time complexity is measured in milliseconds (ms).

Table 4 reports the performance rustles of the time complexity of normal or abnormal classification of VAG signal images. As shown in the tabulated results, the proposed technique outperforms well than the other two methods. Let us consider the $15 \mathrm{VAG}$ signal images, the time taken to classify the input VAG signal images is $12 m s$ by using the GSBFS-RKLSSVC technique. Similarly, the amount of time consumed by Athavale and Krishnan (2020) and Sharmaa and Acharya (2018) are 14ms and 16ms respectively based on the similar count of VAG signal image samples. The overall ten results of proposed technique are compared to existing results and noticed that GSBFS-RKLSSVC technique minimizes time complexity by $10 \%$ and $17 \%$ as compared to Athavale and Krishnan (2020) and Sharmaa and Acharya (2018), respectively.

Figure 8 portrays results of time complexity with number of VAG signal images. As shown in figure 8 , the time complexity is increased for all the methods while increasing the input VAG signal images. But comparatively, the proposed GSBFS-RKLSSVC technique consumes lesser time complexity than the others. This is due to the application of the Greedy mutual informative regressed sequential backward selection algorithm. The number of features is obtained from the VAG signal image. Extracted features are given to the sequential backward selection algorithm to select the best features for classification and discard the remaining features based on the mutual information gain. The dichotomous logit regression is applied to analyze 
and evaluate the mutual information between the features and select the more relevant features. Then the classification is presented with selected features to find normal or abnormal VAG signal image with minimum time.

\section{Conclusion}

In the proposed article, a GSBFS-RKLSSVC technique is employed for VAG signal analysis. The main aim of the GSBFS-RKLSSVC technique is to detect the knee joint pathology based on the VAG signal image analysis through two different processes namely feature selection and classification. The GSBFS-RKLSSVC technique initiates the feature selection process using a Greedy mutual informative regressed sequential backward selection algorithm. The dichotomous logit regression is applied in the dichotomous logit regression for feature evaluation based on the mutual information. The regression function returns the best features and it is used for classification. This increases classification and reduces time complexity. Then GSBFSRKLSSVC technique performs the classification based on the Radial basis kernelized least square support vector classifier. Finally, the kernel-based classification technique identifies the normal and abnormal VAG signal images with higher accuracy and lesser time consumption. The comprehensive experimental assessment is carried out with VAG signal images. The quantitative evaluation is done and the results proved that the GSBFS-RKLSSVC technique

achieves higher accuracy of VAG signal classification with lesser time and error rate when compared to other related works.

\section{Compliance with Ethical Standards}

Ethical approval This article does not contain any studies with human participants or animals performed by any of the authors.

Funding This study was not funded by any funding agency

Conflict of interest The authors declare that they have no conflict of interest.

Informed Consent Not applicable

Authorship Contribution Both the authors equally contributed 


\section{References}

Adam Ł A, Froń A, Bączkowicz D Szmajda M (2020) Vibroarthrographic Signal Spectral Features in 5-Class Knee Joint Classification. Sensors 20:1-15

Andersen RE, Arendt-Nielsen L, Madeleine P (2018) Knee joint vibroarthrography of asymptomatic subjects during loaded flexion-extension movements. Med Biol Eng Comput 56:2301-2312

Athavale Y, Krishnan S (2020) A telehealth system framework for assessing knee-joint conditions using vibroarthrographic signals. Biomed Signal Proces Cont 55:1-9

Befrui N, Elsner J, Flesser J, Huvanandana J, Jarrousse O, Le TN, Müller M, Schulze WHW, Taing S, Weidert S (2018) Vibroarthrography for early detection of knee osteoarthritis using normalized frequency features. Med Biol Eng Comput 56:1499-1514

Fredo ARJ, Josena TR, Palaniappan R, Mythili A (2017) Classification of Normal And Knee Joint Disorder Vibroarthrographic Signals using Multifractals and Support Vector Machines. Biomed Eng Appl Basis Comm 29(3):1-9

Gong R, Hase K, Goto H, Yoshioka K, Ota S (2020) Knee osteoarthritis detection based on the combination of empirical mode decomposition and wavelet analysis. J Biomech Sci Eng 15(3):1-12

Kalo K, Niederer D, Sus R, Sohrabi K, Groß V, Vogt L (2020a) Reliability of Vibroarthrography to Assess Knee Joint Sounds in Motion. Sensors 20:1-9

Kalo K, Niederer D, Sus R, Sohrabi S, Groß V, Vogt L (2020b) The detection of knee joint sounds at defined loads by means of vibroarthrography. Clin Biomech 74:1-7

Kręcisz K, Bączkowicz D (2018) Analysis and multiclass classification of pathological knee joints using vibroarthrographic signals. Comput Meth Prog Biomed 154:37-44

Łysiak A, Froń A, Bączkowicz D, Szmajda M (2019) The new descriptor in processing of vibroacoustic signal of knee joint. IFAC-PapersOnLine, 52(27):335-340

Madeleine P, Andersen RE, Larsen JB, Arendt-Nielsen L, Samani A (2020). Wireless multichannel vibroarthrographic recordings for the assessment of knee osteoarthritis during three activities of daily living. Clin Biomech 72:16-23

Nalband S, Sreekrishna RR, Prince AA (2016). Analysis of Knee Joint Vibration Signals using Ensemble Empirical Mode Decompositio. Proc Comput Sci 89:820-827

Nalband S, Valliappan CA, Prince AA, Agrawal A (2018) Time-frequency based feature extraction for the analysis of vibroarthographic signals. Comput Elect Eng 69:720-731

Nouma BB, Mitiche A, Mezghani N (2019) A Sample-Encoding Generalization of the Kohonen Associative Memory and Application to Knee Kinematic Data Representation and Pathology Classification. Appl Sci 9(9):1-15 
Ołowiana E, Selkow N, Laudner K, Puciato D, Bączkowicz D (2020) Vibroarthrographic analysis of patellofemoral joint arthrokinematics during squats with increasing external loads. BMC Spor Sci Med Rehab 12:1-9

Samani A, Andersen RE, Arendt-Nielsen A, Madeleine P (2020) Discrimination of knee osteoarthritis patients from asymptomatic individuals based on pain sensitivity and knee vibroarthrographic recordings. Physiol Measur 41(5):1-29

Sharmaa M, Acharya UR (2018) Analysis of knee-joint vibroarthographic signals using bandwidth-duration localized three-channel filter bank. Comput Elect Eng 72:191-202

Shieh C-H, Tseng C-D, Chang L-Y, Lin W-C, Wu L-F, Wang H-Y, Chao P-J, Chiu C-L, Lee T$\mathrm{F}$ (2016) Synthesis of vibroarthrographic signals in knee osteoarthritis diagnosis training. BMC Res Notes 9:1-10

Yiallourides C, Naylor PA (2020) Time-Frequency Analysis and Parameterisation of Knee Sounds for Non-invasive Detection of Osteoarthritis. IEEE Trans Biomed Eng 1-13. https://doi.org/10.1109/TBME.2020.3024285

Zheng Y, Wang Y, Liu J, Jiang H, Yue Q (2020) Knee joint vibration signal classification algorithm based on machine learning. Neural Comput Appl 1-11 
Figures

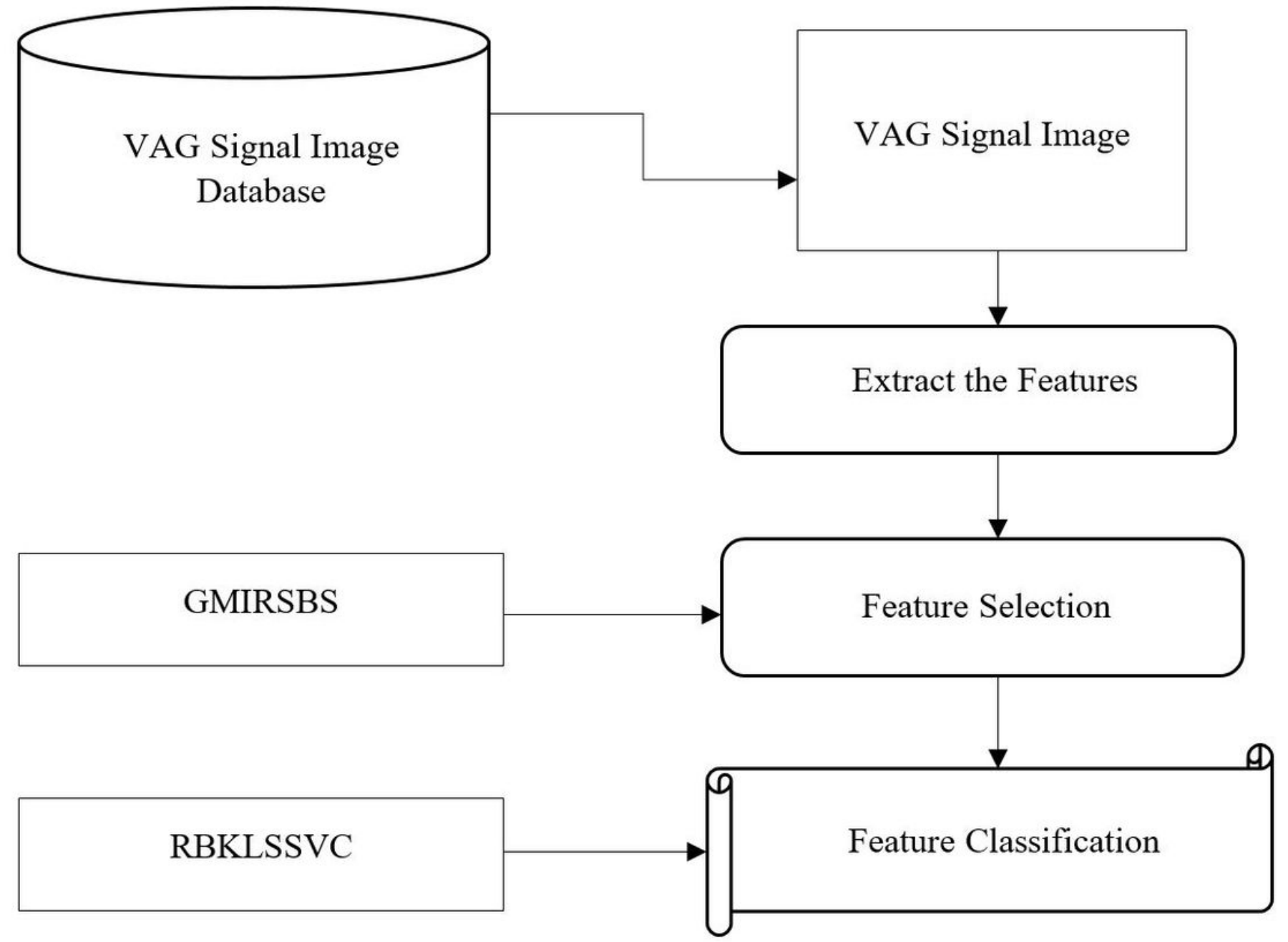

Figure 1

Architecture of the Proposed GSBFS - RKLSSVC Technique 


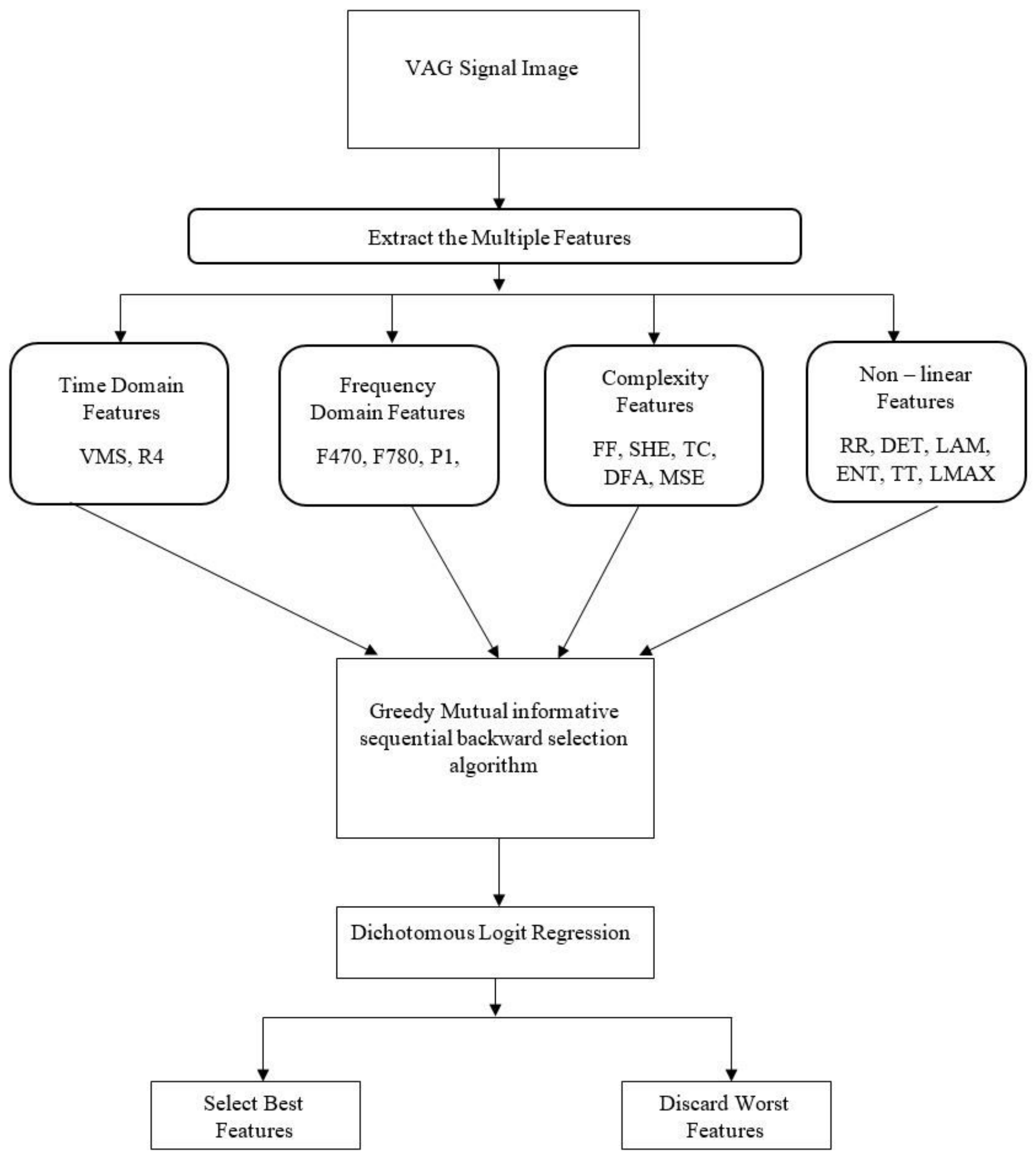

Figure 2

Flow Process of GMIRSBS Algorithm 


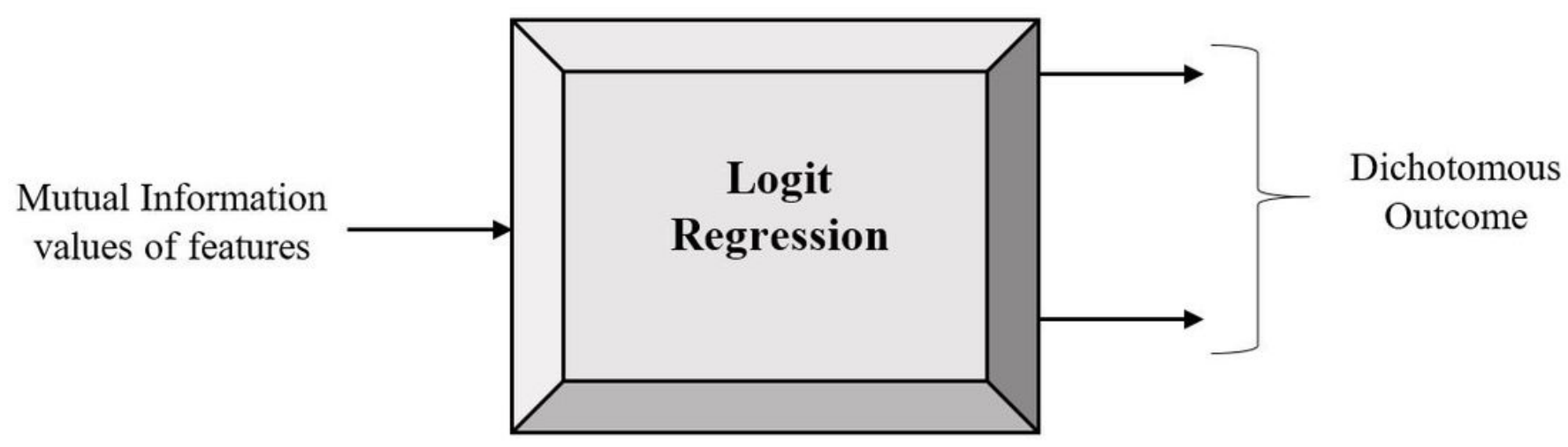

Figure 3

Dichotomous Logit Regression

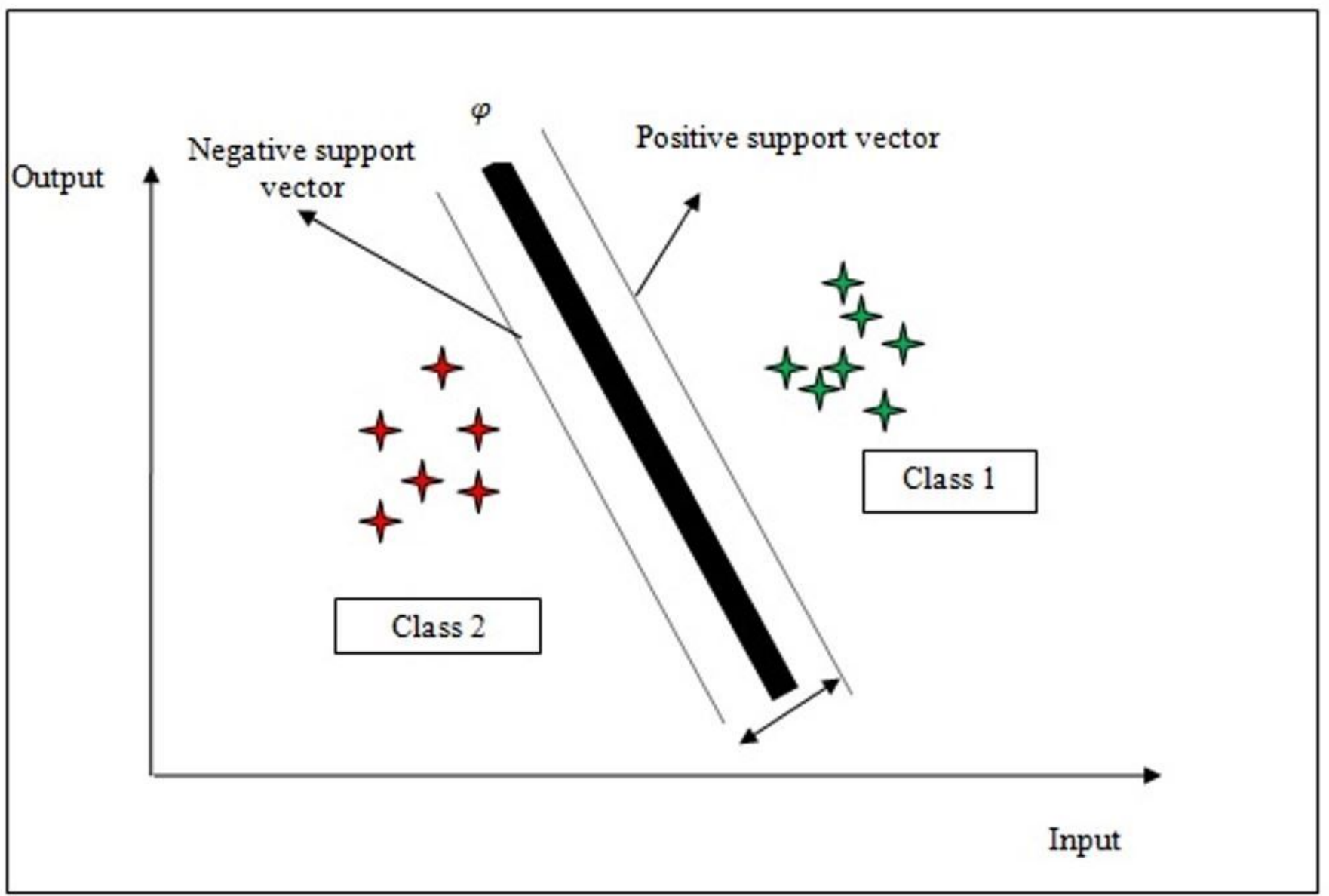

Figure 4

Construction of RBKLSSVC 


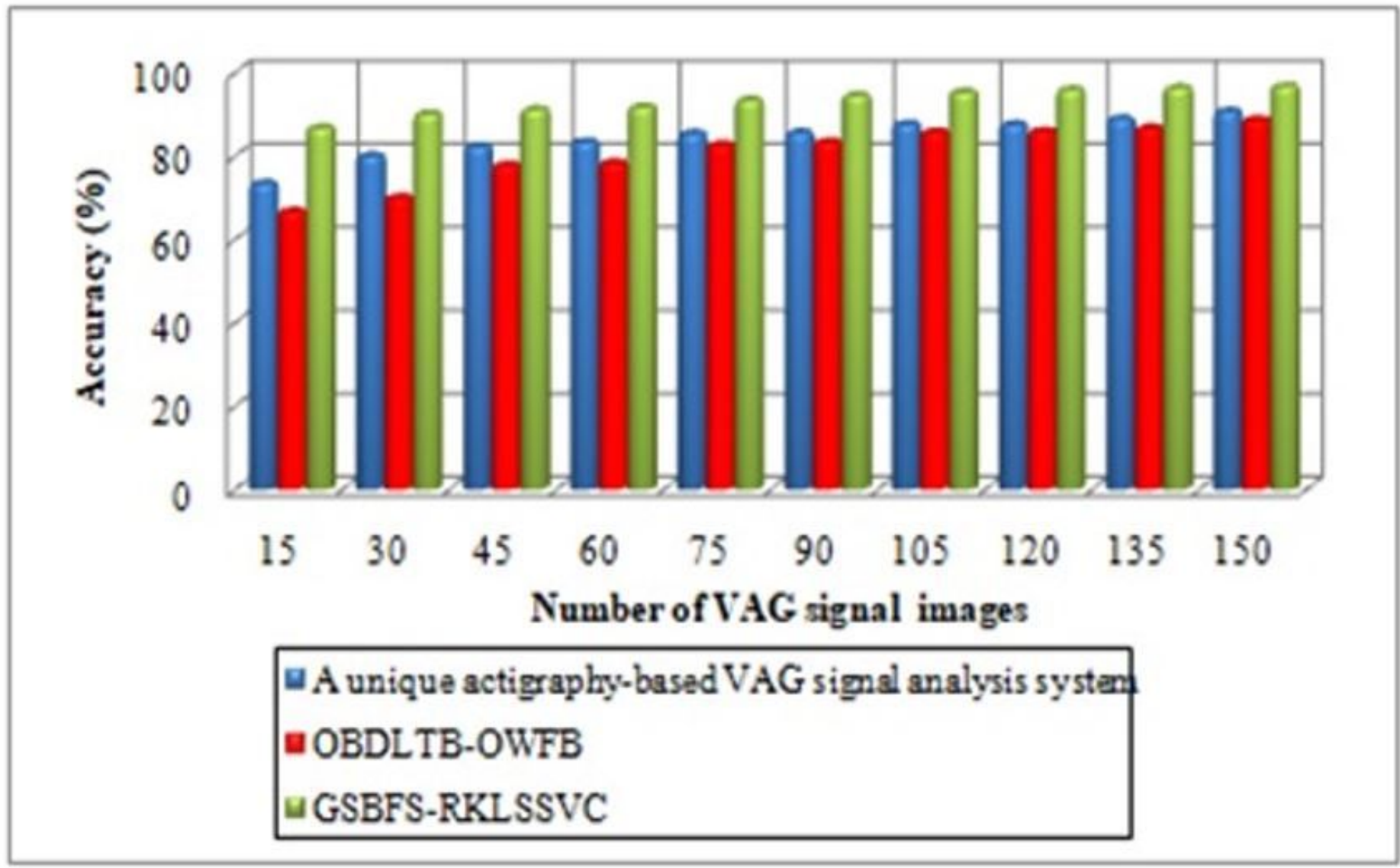

Figure 5

Accuracy Versus the number of VAG Signal Images 


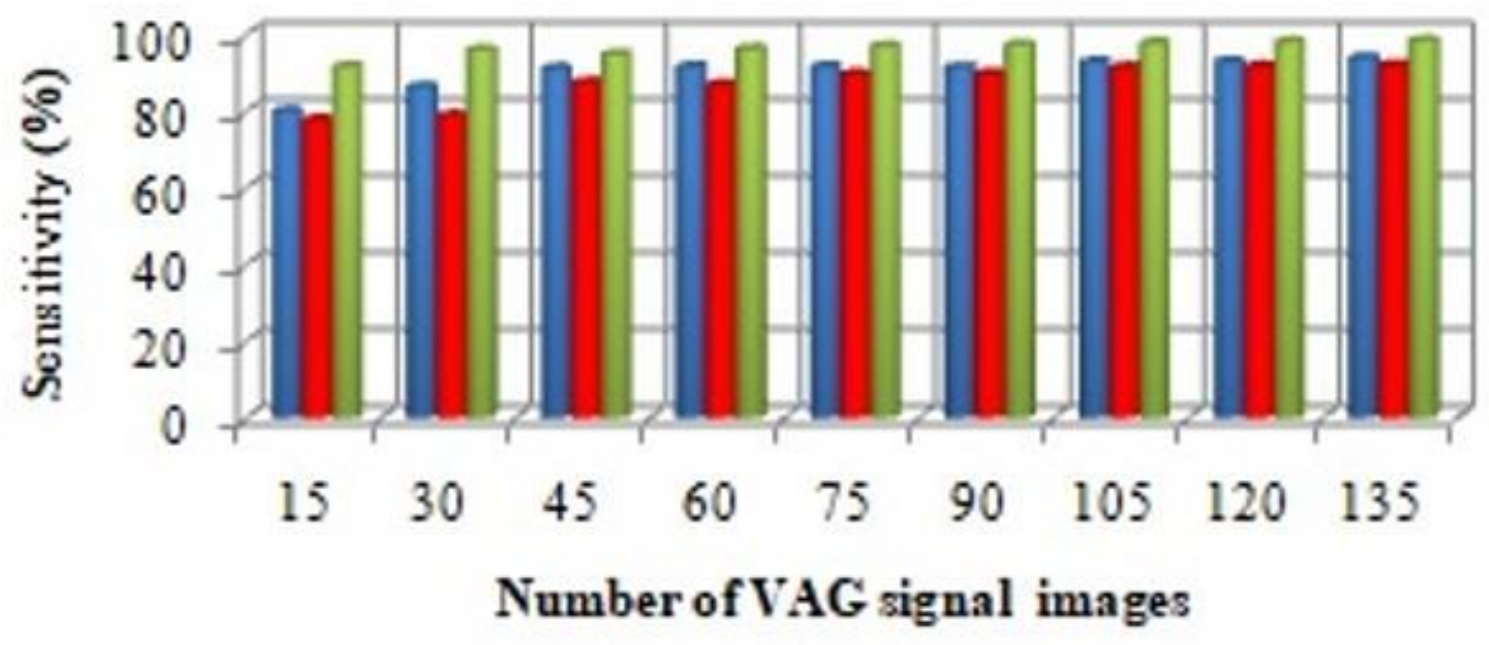
- A Unique Actigraphy based VAG Analysis
- OBDLTB-OWFB
ॠGSBFS-RKLSSVC

Figure 6

Sensitivity Versus the number of VAG Signal Images 


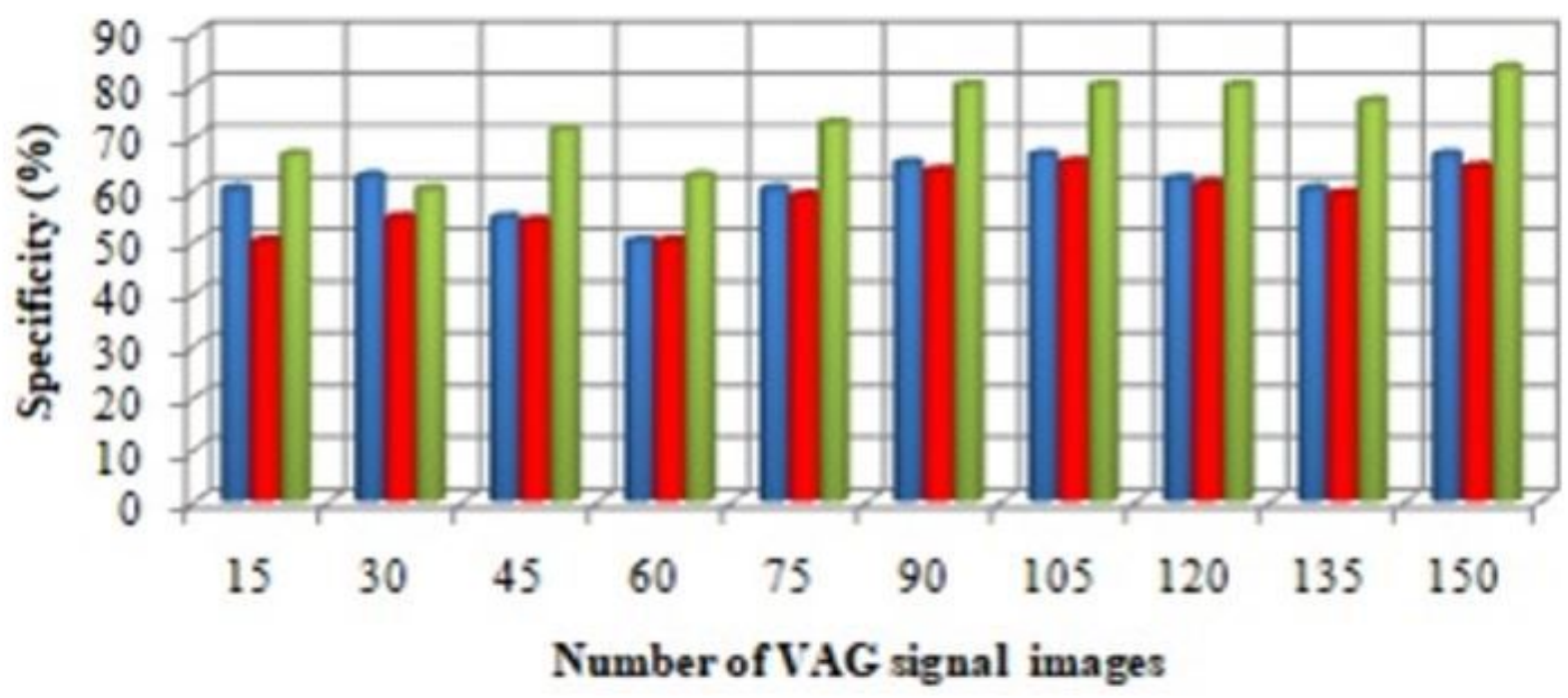

- A Unique Actigraphy based VAG Analysis

- OBDLTB-OWFB

= GSBFS-RKLSSVC

Figure 7

Specificity versus number of VAG Signal Images

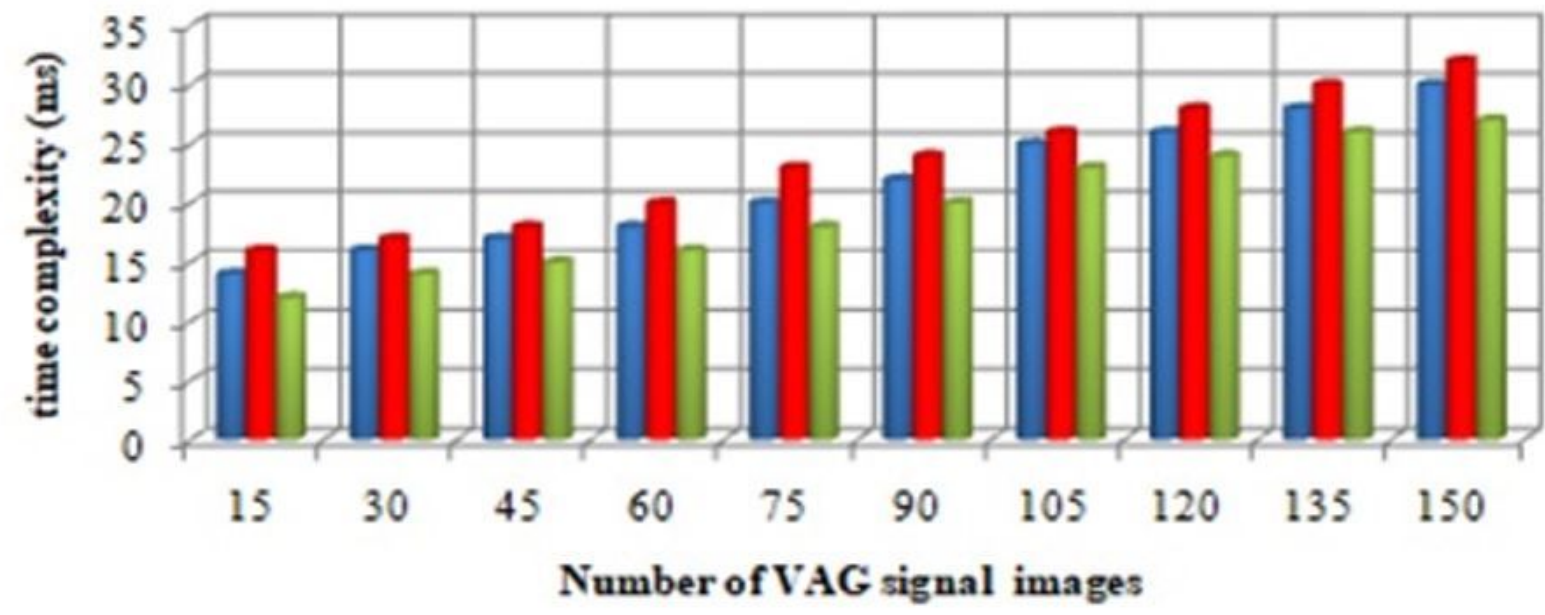

- A Unigue Actigraphy based VAG Analysis

- OBDLTB - OWFB

a GSBFS - RKLSSVC 
Figure 8

Time Complexity Versus the number of VAG Signal Images 